\title{
Kernos
}

Revue internationale et pluridisciplinaire de religion grecque antique

9 | 1996

Varia

\section{Le Dromos au coeur de l'agora de Sparte. Les dieux protecteurs de l'éducation en pays dorien}

Points de vue nouveaux

\section{Patrick Marchetti}

\section{(2) OpenEdition}

Journals

Édition électronique

URL : http://journals.openedition.org/kernos/1165

DOI : 10.4000/kernos. 1165

ISSN : 2034-7871

Éditeur

Centre international d'étude de la religion grecque antique

\section{Édition imprimée}

Date de publication : 1 janvier 1996

ISSN : 0776-3824

Référence électronique

Patrick Marchetti, « Le Dromos au coeur de l'agora de Sparte. Les dieux protecteurs de l'éducation en pays dorien », Kernos [En ligne], 9 | 1996, mis en ligne le 21 avril 2011, consulté le 02 mai 2019. URL: http://journals.openedition.org/kernos/1165; DOI : 10.4000/kernos.1165 


\title{
Le « Dromos » au coeur de l'agora de Sparte.
}

\author{
Les dieux protecteur's de l'éducation en pays dorien.
}

\author{
Points de vue nouveaux
}

Les grandes agoras grecques, du Péloponnèse notamment, sont dans l'ensemble très mal connues. Nous sommes condamnés, pour pallier notre ignorance, à exploiter au maximum la Périégèse de Pausanias, en nous efforçant tantôt d'en tirer une identification pour les ruines que révèle la fouille ${ }^{1}$, tantôt de reconstituer des «itinéraires » qui aboutissent à des constructions abstraites $^{2}$. Le plus souvent chaque site est traité comme une entité distincte au sein de laquelle chaque édifice consacré à tel dieu ou déesse est considéré d'abord pour lui-même, comme si chaque cité constituait un ensemble spécifique. Pour progresser dans l'analyse et la reconstitution historique, il convient de rompre cette problématique trop étroite en intensifiant les comparaisons, en rapprochant par exemple la description de l'agora de Sparte chez Pausanias - dont on sait qu'elle est particulièrement détaillée - de celle que le Périégète a donnée d'Argos, en tirant parti des renseignements exceptionnels livrés par les fouilles de ce dernier site pour mettre en perspective quelques éléments fondamentaux de toute agora, qui nous permettent de remonter aux temps anciens des fondations. Il n'est pas de meilleure démarche si l'on veut faire sa place à un site comme Sparte, dans un colloque consacré à la religion des cités-états des époques archaique et

1 Les exemples de Corinthe et de l'Isthme sont à cet égard éloquents. On a peine à suivre, depuis G. Roux, Pausanias en Corintbie, 1958, toutes les remises en question auxquelles prêtent les identifications.

2 C'est ici l'exemple de Sparte qui est le plus caractéristique. Les propositions d'attribution sont nombreuses depuis N.E. CROSBY, The Topograpby of Sparta, in AJA, 8 (1893), p. 335373, jusqu'à C.M. STIBBE, Beobacbtungen zur Topograpbie des antiken Sparta, in BABesch, 64 (1989), p. 61-99, en passant par D. MUSTI \& M. TORELLI, Pausania. Guida della Grecia. III, 1992, not. p. L-LI et le commentaire passim. Un bel exemple de la fragilité des hypothèses est fourni par

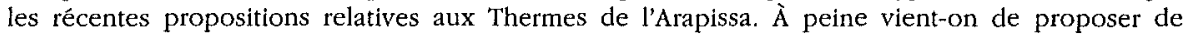
reconnaître dans ces ruines situées à $500 \mathrm{~m}$ du théâtre le fameux gymnase d'Euryklès, cité par PAUSANIAS, III, 14,6 - cf. O. PALAGIA, in S. WALKER \& A. CAMERON (éds), The Greek Renaissance in the Roman Empire, 1989 , p. 122-129 - que M. TORELL, in M. GNABE (éd.), Stips votiva. Papers presented to C.M. Stibbe, 1991, p. 227 sq., propose d'y localiser un Plataniste construit à l'image du "Théâtre Maritime " de la Villa Hadriana de Tivoli. Pour un premier essai d'interprétation, dans la ligne de ce que nous proposons ici, voir P. MARCHETTI, $\mathrm{K}$. KOLOKOTSAS, le nymphée de l'agora d'Aigos, 1995, p. 207-220. 
classique. On posera en tout cas comme hypothèse de travail qu'exception faite des cultes nouveaux introduits sous l'Empire, le tissu religieux de Sparte n'a pas été substantiellement altéré de l'époque archaïque à l'époque impériale.

\section{L'Hélos de Sparte et le Dromos}

La description de la ville de Sparte chez Pausanias ${ }^{3}$ est touffue. Il est difficile d'en jalonner les nombreux détours et le risque est grand de vouloir $y$ mettre plus d'ordre et de raison qu'il ne s'y trouve en réalité, en se laissant abuser par l'apparente logique d'un texte structuré selon des itinéraires bien nets qui auraient pris pour cibles successivement le théâtre, "l'agora », le Cboros, l'Aphétaïde, le Dromos et le Plataniste, autant de lieux que la lecture de Pausanias invite à imaginer comme distincts et éloignés les uns des autres. Les identificiations sont d'autant plus problématiques que le site est encore largement muet ${ }^{4}$ : seuls le sanctuaire d'Artémis Orthia d'un côté et, de l'autre, l'acropole avec le théâtre qui s'y accroche et le temple d'Athèna Chalchioukos qui en occupe l'un des plateaux offrent des points de repère indiscutables. Mais les questions surgissent dès qu'il s'agit de préciser le rapport qui pouvait exister entre la "stoa romaine ${ }^{5}$ qui limite au sud-est le plateau oriental de l'acropole, et « l'agora » de Pausanias; l'indigence notamment explique que se multiplient les hypothèses à propos de l'imposante structure circulaire relevée dans le prolongement de la stoa ${ }^{6}$, tandis que l'une des dernières trouvailles -

III, 11, 1 - 18, 5. Précisons d'emblée que le lecteur ne doit pas s'attendre à trouver ici une nouvelle tentative de reconstituer les itinéraires du Périêgète. En exploitant le témoignage d'inscriptions d'époque impériale, il nous suffit pour l'instant de saisir de la description de Pausanias les renseignements objectifs qu'elle livre et d'en exploiter les indications pour comprendre l'organisation de l'espace public, autour de certains dieux spécifiques dont la présence sacralise les lieux voués à la vie civique.

4 Pour une présentation récente du site, nous renvoyons à l'ouvrage de P. CARTLEDGE \& A. SPAWFORTH, Hellenistic and Roman Sparta, 1989, p. 213-225, et au bilan des foulles de Sparte dans G.B. WAYWELl \& J.J. Wilkes, Excavations at Sparta: The Roman Stoa, 1988-1991. Part 2, in $A B S A, 89$ (1994), p. 379-384, où l'on trouvera toute la bibliographie utile.

5 Où les fouilles ont repris entre 1988 et 1991, cf. les rapports de G.B. WAYWELL \& J.J. WILKES, in $A B S A, 88$ (1993), p. 216-286 et 89 (1994), p. 377-432. On notera qu'à l'époque de Pausanias, cette stoa était toute récente, puisque l'on en date la construction entre 125 et 150 ap. J.-C.

6 Parce qu'il est rond, ce soubassement a été rapproché de la mention chez Pausanias d'un

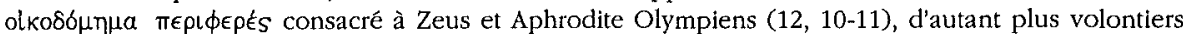
qu'une base de statue en occupe le centre, mais on a songé aussi au Cboros ou à une orchestra (comme F. KOLB, Agora und Theater, 1981 [Arcbäologiscbe Forscbungen, 9], p. 80 et 111), à un petit théâtre (R. MARTIN, Rech. sur l'agora grecque, 1951 [BEFAR, 174], p. 233-235), à la Skias (F. ROBERT, Thymélé, 1939 [BEFAR, 147], p. 113 sq.), à la base de la statue du Démos (CROSBY, art. cit. [n. 2], p. 342). Le caractère chthonien des divinités de l'endroit serait pourtant établi, $c f . B C H, 89$ (1965), p. 717-723. Pour les fouilles récentes qui entraînent une hypothèse nouvelle (cénotaphe de Brasidas), $c f . A B S A, 89$ (1994), p. 418-419. Il est surprenant que le témoignage de DIOGÈNE LAËRTE,, I, 115, relatif aux consécrations d'Épiménidès, ne soit jamais versé au débat lorsqu'il s'agit de

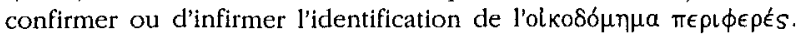


les puissants murs de terrasse en bel appareil exhumés à proximité ${ }^{7}$ - pique la curiosité plus qu'elle n'offre un ancrage à la localisation d'un des très nombreux édifices signalés par le Périégète. Et les fouilles récentes de l'«agora romaine » et du théâtre par l'École anglaise, pour importants qu'en soient les apports nouveaux sur l'histoire de ces édifices, n'ont rien livré encore - à notre connaissance - pour la solution des problèmes topographiques. Dans ces conditions la lecture de l'espace public de Sparte serait désespérante sans les indications fournies par quelques inscriptions d'époque impériale qui autorisent à recentrer autour d'un même quartier certains des sanctuaires les plus importants de la cité, offrant ainsi une clé majeure pour l'interprétation des lieux publics. La révision qui s'ensuit amène la découverte d'une structure urbaine compacte, cohérente et singulièrement organisée autour d'un complexe stratégique, le Dromos, lieu éminent où l'on sacralisait l'intégration des jeunes à la cité.

\subsection{Les inscriptions d'époque impériale et l'Hélos}

L'abondance des cultes à l'époque impériale et les retombées sociales de leur exercice expliquent qu'ils aient été regroupés dans des prêtrises exercées à titre prestigieux et onéreux par les représentants des quelques familles de l'oligarchie lacédémonienne d'époque impériale ${ }^{8}$. Les services constitutifs de ces prêtrises à vie et le plus souvent héréditaires étaient, pour la plupart, relatifs à des dieux dont les sanctuaires étaient contigus. Ainsi en va-t-il notamment pour la série des cultes associés de l'Hélos, en l'occurrence un quartier de Sparte $^{9}$ localisé dans une ancienne limnè. Quelques textes épigraphiques sont particulièrement intéressants.

\section{La prêtrise commune d'Aphrodite Enoplios, d'Asclépios Schoinatas et des Moires}

Les sanctuaires des Moires «Lacheseis», d'Aphrodite «Enoplios» et d'Asclépios «Scboinatas» sont explicitement localisés év Tọ "E inscription honorifique ${ }^{10}$. Il apparaît d'emblée que le regroupement des trois

Cf. $A B S A, 89$ (1994), p. 383.

8 Pour le contexte historique, nous renvoyons à CARTLEDGE \& SPAWFORTH, op. cit. (n. 4).

9 Que l'on a confondu - de manière incompréhensible - avec la ville de même nom du littoral laconien, qui était en ruines à l'époque romaine comme le signalent et STRABON, VIII, 363 et PAUSANIAS, III, 22, 3. Là-dessus, $c f$. MARCHETTI \& KOLOKOTSAS, op. cit. (n. 2), p. 211-212, n. 46. Nous présentons ici les témoignages relatifs à ces difficiles questions dans une perspective toute différente de celle du Nympbée de l'agora d'Argos, en partant des inscriptions pour aller à Pausanias et non l'inverse. Les conclusions que l'on peut tirer de cette confrontation entre le texte de Pausanias et les inscriptions sont si importantes que les deux modes d'approche s'imposent comme démarches complémentaires. Nous insistons aussi davantage ici sur les dieux associés dans l'Hélos.

$10 I G, \mathrm{~V} 1,602$. Outre les cultes d'Artémis Orthia et d'Artémis « en Pleiais », le texte signale que

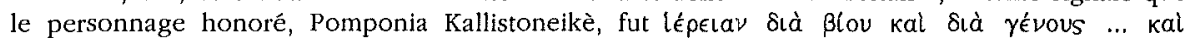

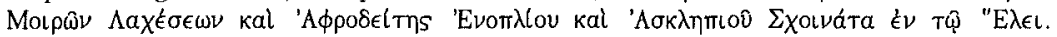


divinités dans un service religieux commun n'est pas fortuit. On ne peut, en effet, s'étonner de trouver les Moires en compagnie d'Aphrodite, à songer que les théogonies orphiques, mais aussi le sage Épiménidès ${ }^{11}$ font des unes et de l'autre des scurs issues d'Ouranos ou de Cronos $^{12}$ et alors que l'Aphrodite $\omega \pi \lambda\left\llcorner\sigma \mu \epsilon \nu \eta^{13}\right.$ tout particulièrement est avant tout la déesse « ouranienne ${ }^{14}$. La conjonction d'Asclépios Schoinatas et d'Aphrodite n'est pas davantage fortuite : on n'a guère de peine à retrouver dans l'épiclèse Schoinatas une détermination liée au quartier "Eגos où se localise le sanctuaire ${ }^{15}$; or, l'épiclèse

11 Autorités auxquelles les Spartiates se réfèrent précisément pour y rattacher la fondation de leurs sanctuaires : Orphée est, encore à l'époque impériale, le fondateur réputé des sanctuaires de Déméter et Corè (PAUS., III, 13, $2 \& 14 ., 5, c f$. infra), tandis que les Spartiates disputaient aux Argiens l'honneur d'abriter la tombe d'épiménidès située à côté d'Hermès Agoraios, donc dans le voisinage immédiat du sanctuaire des Moires et d'Aphrodite Enoplios (ibid., III, 11, 11, cf. infra). C'est aussi à Épiménidès que les Spartiates attribuaient la construction de l'édifice rond voué à Aphrodite et Zeus Olympiens ( 1 bid., III, 12,11), autre rapport entre Épiménidès et Aphrodite.

12 Cf. O. KERN, Oppbiconum Fragmenta, 1922, p. 137 F57 et, pour Epiménidès e.a. H. DIELs \& W. KRANZ, FVS, I, p. 36 F19 et F. JACOBY, FGrH, IIIB 457, p. 391 F7 (Scholie à SOPHOCLE, CEd. Col. 42). Chez Hésiode, les Moires sont filles de Nuit (Th., 217-219) et, subsidiairement, de Zeus et Thémis (Tb., 904-906, cf. T. GANTZ, Early Greek Myth, 1994, p. 7-8), La filiation par Zeus et Thémis est secondaire et sans incidence pour la reconstitution des cosmologies authentiques où s'inscrivent les Moires : le fait de les voir figurer à côté des Heures, comme filles de Zeus et Thémis, et avant les Grâces - filles de Zeus et Eurynomè - montre bien que les Moires viennent en surnombre parmi ces «enfants de Zeus ». La première filiation hésiodique - les Moires sont filles de Nuit - rejoint en fait la cosmologie d'Épiménidès pour qui Air et Nuit sont les deux principes premiers (H. DIELS \& W. KRANZ, op. cit, p. 33 F5 et F. JACOBY, op. cit., p. 390 F4, cf. G. COLLI, La Sagesse grecque, II, 1978 [1991 pour la tr. franç.], p. 58-61 F8[B3]). Que les Moires descendent de Cronos, comme chez Épiménidès, ou d'Ouranos, comme dans le fragment 57 des Orphica, est sans conséquence ici : dans les deux cas leur naissance est associée à Aphrodite qui dans les deux filiations mérite son épiclèse d'« Ouranienne ». Et l'on ne peut que rapprocher cette épiclèse des

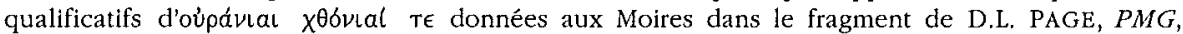
p. $532 \mathrm{n}^{\circ} 1018$, dont une partie au moins peut être attribuée à Euripide ( $c f$. A. NAUCK, TGF, p. 555 $\mathrm{F} 620$, à compléter par p. XX $\mathrm{n}^{\circ}$ 3). Le sort des Moires qui de filles d'Ouranos sont devenues filles de Zeus est similaire à celui des Muses, chantées par Mimnerme (PAUS., IX, 29, 4 = M.L. WEST, Iambt $e$ Elegi Graeci, $\mathrm{II}^{2}$, p. 89, F13), qui distinguait des Muses "plus anciennes », filles d'Ouranos, et des Muses «plus jeunes», filles de Zeus, tandis qu'une scholie à Apollonios de Rhodes (cf. G. COLLI, op. cit., I, 1977 [1990 pour la tr. franç.], p. 297) nous apprend que chez Musée les mêmes Muses anciennes sont filles de Cronos - qui prend donc, ici comme pour les Moires, la place d'Ouranos! - et les plus récentes filles de Zeus et de... Mnémosyne. Sur toutes ces traditions, en apparence seulement contradictoires, cf. M.L. WEST, The Orphic Poems, 1983.

13 Sur l'épiclèse donnée par Pausanias et la variante d'un manuscrit, $c f$. V. PIRENNEDELFORGE, L'Aphrodite grecque, 1994, p. 211.

14 Comme à Cythère, $c f$. PAUS, III, 22, 1 (Sur Cythère et son Aphrodite, nous renvoyons à l'exposé détaillé de PIRENNE-DELFORGE, op. cit. [n. 13], p. 217-226). Et peut-on imaginer que l'Aphrodite «armée» de Sparte puisse être différente de la Cythéréenne? C'est évidemment le rapport très étroit entre les Moires et Aphrodite, notamment par l'intermédiaire de leurs pères communs, qui rend compte de la singularité signalée par Pausanias, qui avait lu à Athènes sur le

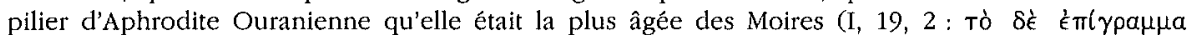

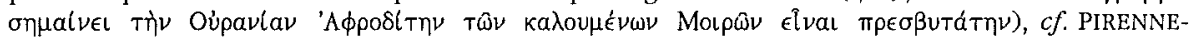
DELFORGE, ibid., p. 48.

15 Le passage d'Alexis de Samos cité par Athénée, Deipnosopbistae, XIII, 572, ne laisse aucun

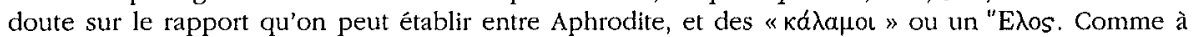
Kalamoi se trouvait aussi l'Héraion de Samos, d'après HÉrodoTE, IX, 96, l'Aphrodite "aux 
convient aussi à Aphrodite, qu'on peut appeler $\Sigma \chi$ out à Sparte, implantée à la lisière d'un antique marais ${ }^{16}$. Enfin, la conjonction d'Asclépios et des Moires «Lachéseis » apparaît des plus naturelles à lire l'hymne d'Isyllos ${ }^{17}$, si naturelle même que l'on ne peut tenir pour fortuite la construction du sanctuaire d'Asclépios à côté de celui des Moires. Une telle cohérence invite à approfondir l'examen pour exploiter au mieux toutes les contiguiltés topographiques liées à l'Hélos.

Les sanctuaires d'Asclépios et d'Aphrodite « en armes » sont conjointement évoqués par Pausanias ${ }^{18}$ dans une chaîne topographique qui commence au thêâtre et dont les principaux éléments s'articulent ainsi :

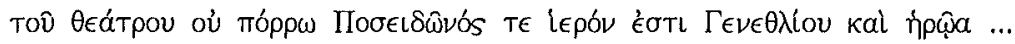

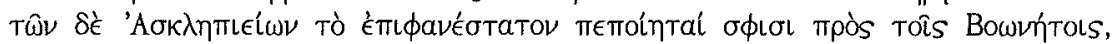

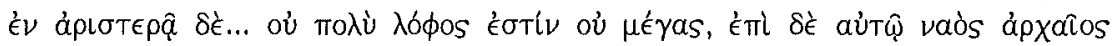

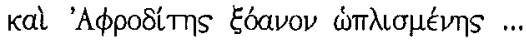

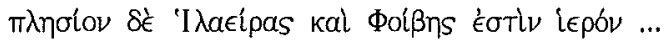

D'autre part, du voisinage immédiat du sanctuaire des Moires et de ceux d'Asclépios Schoinatas et d'Aphrodite Enoplios, nous avons une confirmation péremptoire avec le recoupement qui s'opère autour des Boônéta : il est le premier édifice que rencontre Pausanias $(12,1)$ lorsqu'il atteint l'Aphétaïde en venant « de l'agora », après avoir dépassé le sanctuaire des Moires $(11,11)$; or c'est très précisément iròs tốs Bowvńtous (texte ci-dessus) qu'était érigé l'Asclépieion et le sanctuaire d'Aphrodite « en armes».

Le sanctuaire des Moires figure encore au nombre des curiosités énumérées par Pausanias, au début de sa description de l'agora (11, 9-10), quand le Périégète, en hors-d'œuvre en quelque sorte, évoquait l'endroit appelé Choros pour signaler où móppw, parmi d'autres, les sanctuaires de Zeus Agoraios et d'Athèna Agoraia et conclure une longue litanie de dieux en ajoutant kal

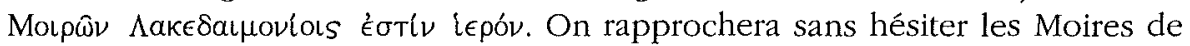
Zeus Agoraios en compagnie duquel on les retrouve à Thèbes ${ }^{19}$. Mais si l'on

marais » de Samos était donc voisine d'Héra, autre indice - parmi beaucoup d'autres - de la proximité d'Aphrodite et d'Héra dans le culte, à Samos comme à Sparte. Nous reprendrons prochainement la question.

16 L'épiclèse est donnée à la déesse chez Lykophron, Alex, v. 832. Cf. W.H. ROSCHER, Lexikon, IV 2, s.v. Schoinatas et Scboineis ; RE, II A 1 (1921), s.v. Schoineis 2; PIRENNE-DELFORGE, op. cit. (n. 13), p. 296.

17 IG, IV, 950, cf. J.U. POWELL, Collectanea Alexandrina, 1925, p. 132-135 (c'est le texte du TLG). On peut y lire entre autres détails intéressants que Lachésis en compagnie des Moires présida à la naissance d'Asclépios, fils d'Apollon Maléate ( $v$. 50-52), et qu'ensuite, une fois né sous de telles auspices, Asclépios s'empressa de porter secours aux Spartiates.

18 III, $15,10-16,1$.

19 PAUS., IX, 25, 4. Thémis y accompagne Zeus Agoraios et les Moires, comme une illustration du fragment de Pindare (Hymnes 1 Puech) où l'on nous montre les Moires conduisant Thémis à Zeus. Pour d'autres associations Zeus (Moiragetes) - Moires, of. LIMC, VI (1992), s.v. Moirai, p. 638 (S. DE ANGELIS). 
n'était pas convaincu par la nécessité de cette contiguïté - ainsi en tenant que

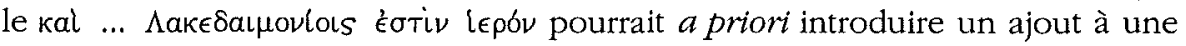
liste par ailleurs cohérente et ne pas nécessairement avoir une portée topographique stricte -, une autre mention du sanctuaire des Moires ${ }^{20}$ après l'évocation d'un Hermès Agoraios lève toute ambiguïté, car ce dernier en répondant à Zeus Agoraios et à Athèna Agoraia nous interdit de détacher la première mention des Moires des autres dieux Agoraioi. La localisation $\epsilon \nu$ T $\hat{\varphi}$ "E $\lambda \epsilon \iota$ du sanctuaire des Moires entraîne dès lors celle du Cboros - et de tous les dieux qui lui sont associés, notamment les Agoraioi - dans ce même quartier, qu'on cherchera du côté du théâtre puisque le sanctuaire des Moires, parce qu'il est associé à ceux d'Aphrodite Enoplios et d'Asclépios Scboinatas, doit se trouver

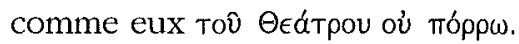

La localisation sur l'agora du sanctuaire des Moires - et donc aussi de l'Hélos - ne fait par ailleurs non plus aucun doute comme il résulte d'un double recoupement. D'une part, la mention des Moires clôt l'énumération des dieux agoraiot situés du côté du Cboros, lieu par lequel Pausanias entamait sa

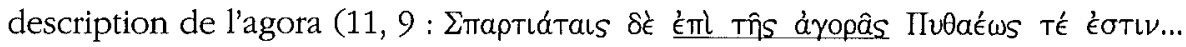

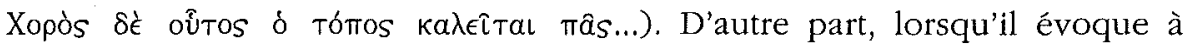
nouveau le sanctuaire des Moires, il le fait juste avant de «quitter l'agora » par

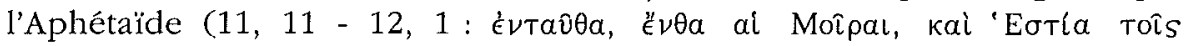

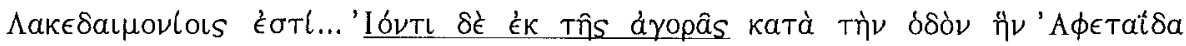

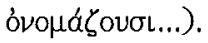

Nous pouvons ainsi conclure que l'Asclépieion et les sanctuaires d'Aphrodite Enoplios et des Moires, proches du théâtre, se trouvaient tout à la fois sur l'agora, près du Cboros $^{21}$ et au voisinage immédiat de l'Aphétaïde ${ }^{22}$.

20 PAUS., III, 11, 11. Dans ce second passage, l'évocation des Moires est insérée entre la mention des Epboreia et d'Hestia. Le rapport de l'un à l'autre est si étroit que nous ne pouvons hésiter à conclure que nous sommes bien là au coeur de la cité, du côté de ces édifices énumérés

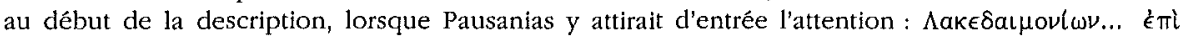
Tîs dyopâs (11, 2). Cf. MuSTI \& TORELli, Paisania, III, p. 197-198.

21 Que le choros se situe bien du côté du théâtre, c'est ce que confirme un rapprochement entre Hérodote et Pausanias à propos du lieu des gymnopédies, $c f$. MARCHETTI \& KOLOKOTSAS, op. cit. (n. 2), p. 214-215.

22 Qu'il soit impossible de séparer ces trois derniers éléments, comme on s'est pourtant ingénié à le faire, nous n'en manquons pas d'indices non équivoques. Ainsi, cet autre rapprochement entre deux passages du Périégète, qui interdit d'éloigner l'« Aphétaïde » du sanctuaire des Moires : à côté du sanctuaire des Moires et de la tombe d'Oreste se dresse la statue de Polydore $(11,9)$, or les Boônéta qui sont sur l'Aphétaïde ne sont rien moins que l'antique demeure de Polydore $(12,3)$. 


\section{D'autres dieux de l'Hélos}

D'autres inscriptions de même époque ${ }^{23}$ regroupent dans une autre prêtrise commune les services religieux de Carneios Boikétas, Carneios Dromaios, Poseidon Dômatitès, Héraklès Génarchas, Corè et Téménios, tous également situés év $T \hat{\omega}$ "E $\mathrm{\lambda} \epsilon \mathrm{l}$, tous dieux importants de l'espace public. Ici aussi, les recoupements avec Pausanias se font tout naturellement :

- ainsi, en constatant que le sanctuaire de Corè est mentionné juste avant Carnéios «qu'on appelle Oikétas » $(13,3)$. Confirmation immédiate de ce que les dieux de l'Hélos associés dans la nouvelle prêtrise sont tout aussi voisins les uns des autres que l'étaient les précédents.

- Une autre correspondance obvie ne peut échapper : comme dans l'inscription, le Périégète mentionne à deux reprises Carneios, une première fois identifié comme Oikétas, une seconde fois $(14,7)$ dans un itinéraire qui procède du Dromos, c'est le Carneios Dromaios.

- Les tours et détours que fait Pausanias à partir du Dromos resserrent en des limites étroites sa description ${ }^{24}$ de ce lieu stratégique, qui débutait par le signalement d'une statue d'Héraklès «à laquelle sacrifiaient les Sphaireis »

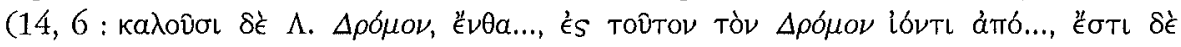

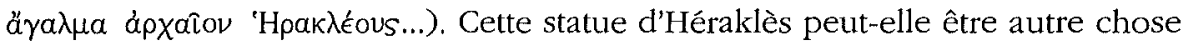
que la représentation de l'Héraklès «Génarchas » associé aux deux Carneios dans la prêtrise commune?

- Enfin, après avoir signalé les Dioscures Aphétériens plantés au début du Dromos et l'bérôon d'Alcon qui en est voisin, Pausanias rencontre, immédiatement avant le Plataniste, Poseidon Dômatitès $(14,7-8)$, l'autre dieu associé aux Carneios, à Corè et à Héraklès dans la prêtrise commune. La présence de ce Poseidon parmi les dieux de l'Hélos rattache cette fois le Plataniste à l'Hélos.

On ne peut donc échapper à l'évidence : la cohérence intrinsèque des services religieux regroupés dans la seconde prêtrise tient à ce que celle-ci regroupe quelques-uns des dieux les plus importants du Dromos, au point que nous pouvons faire de cet espace la partie essentielle de l'Hélos, en prenant soin d'y rattacher aussi le Plataniste dont la description s'articule à la mention de Poseidon Dômatitès. Mais si le Dromos est au cœur de l'Hélos, on est alors amené à recentrer autour de ce lieu tous les sanctuaires des deux prêtrises communes et à tirer en chaîne toutes les conséquences :

$23 I G, \mathrm{~V} \mathrm{1}, 497,1.11-20$, où sont énumérés en une formule dépourvue de toute ambiguỉté les

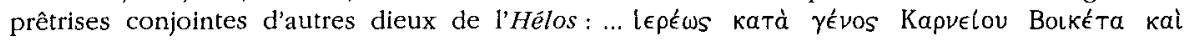

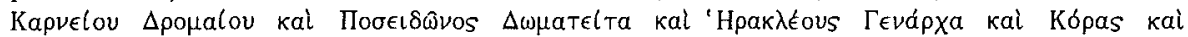

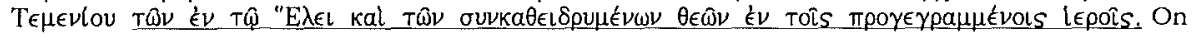
retrouve les mêmes dieux énumérés dans le même ordre en 589, 1. 6-12 et en 608, 1. 2-7.

24 En deux paragraphes (14, 6-7), les références topographiques au dromos sont particulière-

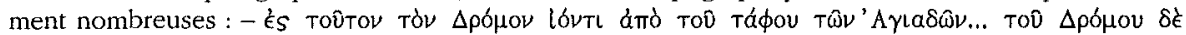

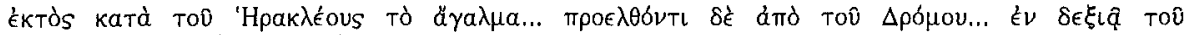

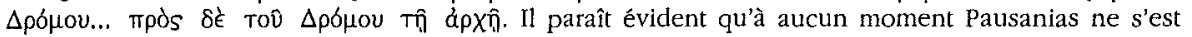
éloigné du Dromos. 
- l'édifice rond de Zeus et d'Aphrodite qui fait face au temple de Corè

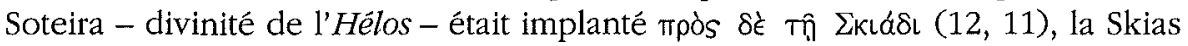

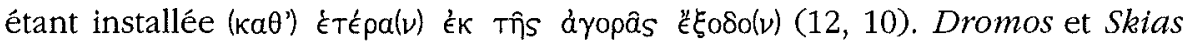
sont donc voisins, tout à côté de «l'agora » de Pausanias.

- Carneios Dromaios, le dieu du Dromos, et Poseidon Dômatitès sont inséparables des Dioscures Apbétériens (14, 6-7), tandis que Carneios Oikétas, lui, n'était « pas éloigné » d'une statue d'Apbétaios qui marquait le départ de la course des prétendants $(13,6)$ - à laquelle est vouée l'Apbétaide $(12,1)$-, dont le départ est proche des Boônéta, l'édifice près duquel se distribuaient les sanctuaires regroupés dans la première prêtrise commune. Dioscures Aphétériens et Aphétaios ne peuvent être séparés de l'Aphétaïde, cette «rue » qui servait à la « course des prétendants » dont Ulysse était le modèle, ni l'Aphétaïde du Dromos, ce qui confirme pleinement les conclusions tirées de la première inscription.

- La cohérence des lieux sort renforcée de la constatation d'une parfaite complémentarité entre les consécrations religieuses rencontrées par Pausanias au cours des deux itinéraires qui l'ont conduit à rencontrer ici le Carneios Oikétas, là le Carneios du Dromos. Il suffit de disposer les notices dans un ordre légèrement différent de l'énumération du texte pour que le parallélisme ressorte aussitôt :

12,10 : près d'une «sortie » de l'agora, SKIAS

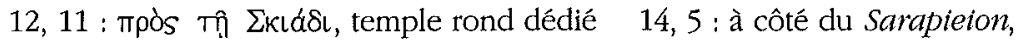
à APHRODITE et ZEUS OLYMPIENS

13, 2 : en face, naos de CORÈ Chthonia consacré par Orphée

\section{3, 3 : CARNEIOS Oikétas}

Près d'Aphrodite et Zeus Olympiens

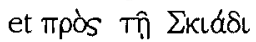

(cf. 12.11 et13.1 : $\pi \lambda \eta \sigma l o v)$

13, 1 : mnèma de CASTOR et tombe d'Idas et LYNCÉE 13, 6 : près du Cameion, la statue d'APHÉTAIOS

trophée de POLLUX sur LYNCÉE.

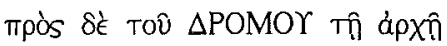
les Dioscures APHÉTÉRIENS

Pour résumer, on peut affirmer que le secteur de la ville appelé Hélos contenait non seulement le Choros, mais aussi le Dromos et le Plataniste, autrement dit que ces trois composantes essentielles de l'espace public spartiate s'interpénétraient autour de l'agora. La confrontation de la description de Pausanias avec les dieux de l'Hélos énumérés dans les inscriptions d'époque impériale nous oblige ainsi à faire table rase des lectures malheureuses

25 Il est très probable qu'il s'agisse de l'Asclépios Scholnatas évoqué plus haut. 
auxquelles Pausanias a donné lieu, et à nous départir de la mauvaise habitude d'éparpiller les curiosités qu'il énumère sur un territoire beaucoup trop vaste. L'essentiel, en réalité, se concentre au pied du théâtre, dans une antique limnè qui correspond à l'Hélos des inscriptions. Dromos et Plataniste sont des prolongements si naturels de «l'agora » de Pausanias qu'on ne peut douter qu'à une époque plus ancienne - quand les édifices y étaient moins nombreux et que les différentes places n'avaient pas encore été délimitées rigoureusement ${ }^{26}$ - ils n'en étaient guère séparés ou distincts. Ainsi lorsque Nabis rassembla toutes ses troupes sur le vaste campus qui coïncidait alors avec le Dromos ${ }^{27}$. D'autre part, si Aphétaios et les Dioscures Aphétériens sont aussi proches du début du Dromos, tirons-en la conviction que l'Aphétaïde s'articule évidemment au Dromos.

\subsection{L'Hélos et l'éducation des éphèbes}

La concentration des espaces les plus caractéristiques de Sparte dans le seul quartier de l'Hélos est d'autant plus intéressante qu'elle concerne en fait tous les lieux publics voués à l'éducation, comme on s'en avise, une fois encore, à la lecture de Pausanias :

- c'est sur le Choros, nous dit Pausanias $(11,9)$, qu'aux gymnopédies, fête célébrée avec beaucoup de zèle, les éphèbes dansent en l'honneur d'Apollon

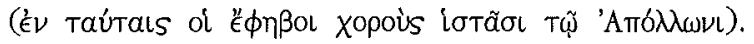

- L'Aphétaïde était symboliquement évoquée comme le lieu de «la course des prétendants » instituée par Icarios à l'imitation de Danaos qui aurait trouvé ce subterfuge pour marier ses filles (12,1-2). Référence mythique particulièrement intéressante, qui confère aux lieux une vocation matrimoniale très nette, dont on retrouve des échos à propos des éphèbes et plus particulièrement des «carneatai » définis comme agamo ${ }^{28}$.

- Le Plataniste, nous dit Pausanias, est l'endroit où les éphèbes ont coutume de se battre.

- Le patron mythique du Dromos spartiate est Héraklès et les souvenirs mythiques de sa lutte contre les fils d'Hippocôon ${ }^{29}$ y sont nombreux, comme autant d'exemples proposés à la jeunesse, laquelle fréquentait à l'époque impériale les gymnases du Dromos. On relèvera la référence explicite aux

26 Notamment avant la construction des gymnases qu'y a vus Pausanias, $c f$. J. DELORME, Gymnasion..., 1960 (BEFAR, 196), p. 73-74.

27 TITE-LIVE, XXXIV, 27, 4-5: cum suspectos quosdam ciutum baberet, eductis in campum omnibus copils - Dromon ipst uocant - positis armis ad contionem uocari iubet Lacedaemontos, atque eorum contiont satellites armatos circumdedit.

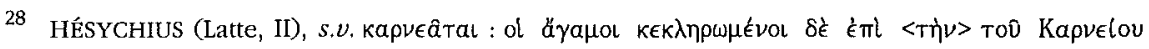

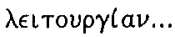

29 Pour une lecture historicisante des luttes d'Héraklès contre les fils d'Hippocôon et de la figure emblématique des Dioscures, les héros par excellence du Dromos, $c f$. C. CALAME, Le récit généalogique spartiate: la représentation mythologique d'une organisation spatiale, in QS, 26 (1987), p. 63-67 notamment. 
entraînements des éphèbes : «les Lacédémoniens appellent Dromos l'endroit où a lieu pour les jeunes gens encore de nos jours l'exercice de la course. En s'y rendant depuis la tombe des Agiades on trouve... une vieille statue d'Héraklès, auquel sacrifient les Sphaireis, ainsi appelle-t-on ceux qui quittent l'éphébie pour devenir adultes » $(14,6)$. Comment ne pas établir de rapport entres les Sphaireis qui deviennent andres, les carneatai qui sont encore agamoi et la " course des prétendants » de l'Aphétaïde ? On se doit aussi de les rapprocher des Dionysiades, qui sont des partheno ${ }^{30}$. Le rapprochement se concrétise une fois de plus sur le terrain puisque c'est du côté du Carneion et de la statue d'Aphétaios $(13,6)$, d'où l'on s'élance pour la course des prétendants, qu'il faut rechercher la «Colline » où se dressait le temple de Dionysos, près duquel est réservé le téménos du héros qui guida le dieu sur la route vers Sparte et auquel «sacrifient les Dionysiades et les Leucippides » $(13,7)^{31}$. Et l'horizon matrimonial très clair trouve encore un écho au sanctuaire d'Aphrodite Héra $^{32}$, situé lui aussi sur une colline (celle de Dionysos ?), où l'on conservait le $x$ oanon «auquel les mères sacrifiaient quand leur fille se mariait » $(13,9: \dot{\epsilon} \pi i$

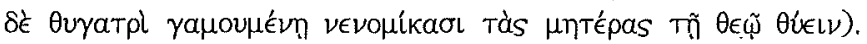

\section{De Sparte à Argos (fig. 1)}

L'évocation de Danaos, à propos de la course de l'Aphétaïde, nous renvoie clairement à Argos, dont l'espace public offre un parallèle rigoureux à la disposition des lieux spartiates telle que nous l'avons reconstituée abstraitement. On y retrouve concentrés en un même lieu - qui est aussi une ancienne limnè, que l'on aurait pu appeler ou que l'on appelait peut-être Hélos comme à Sparte - une piste de danse (l'orchestra de la fig. 1), un gymnase / palestre, une piste de course (le dromos de la fig. 1), et un nymphée. Or, cet espace, lorsqu'il l'a visité, Pausanias l'appelait «agora »33. À cette époque, la

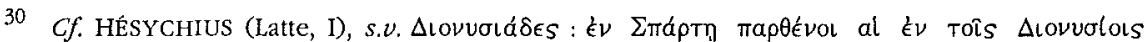

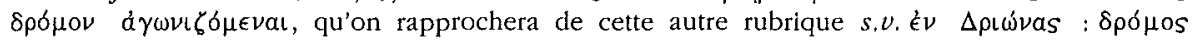

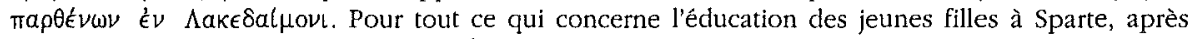
A. BRELICH, Paides e Partbenoi, 1969, voir notamment M.L. NAPOLITANO, Donne spartane e Teknopoita, in Annali delle'Istituto Univ. Orientale, sez. di Arcb. e Storia Ant., 7 (1985), p. 19-49, plus particulièrement p. 28-31. Pour les Dionysiades, les Karneatal et les courses prématrimoniales, cf. M.B. HATZOPOULOS, Cultes et rites de passage en Macédoine, 1994 (Meletèmata, 19), p. 46-53 not.

31 A cette occasion Pausanias précise qu'il y a onze autres Dionysiades, qui participent à un concours de course, «selon une coutume qui leur est venue de Delphes ».

32 Les deux déesses sont tout aussi étroitement associées autour du nymphée, édifice par excellence voué au mariage, $c f$. Le Nymphée de l'agora d'Argos (cité n. 2), p. 237-242, 246. Rappelons qu'à Samos - où l'on connaît une Héra Dromaia (ibid., p. 215-216) - il faut placer Aphrodite dans le quartier appelé Kalamoi ou Hêlos, là où se trouvait le sanctuaire d'Héra (HÉRODOTE, IX, 96). Voir supra, n. 15.

33 Cf. pour une première approche $B C H 117$ (1993), p. 211-223 \& 118 (1994), p. 131-160, et Le Nymphée de l'agora d'Argos, p. 249 sq. 
piste et l'orchestra argiennes étaient probablement tombées en désuétude, comme on peut l'inférer du silence du Périégète, mais la structure de l'espace que les fouilles ont permis de retrouver impose d'établir le parallèle avec Sparte. Et à comparer les deux villes on comprend qu'à Sparte, agora, Dromos, "Aphétaïde", Choros aient pu être singularisés comme autant de lieux distincts d'un ensemble toutefois infrangible, tandis qu'à Argos ils sont tous regroupés en un lieu que Pausanias appelle «agora». Les correspondances entre les structures argiennes et spartiates sont très étroites, les dieux et héros de Sparte ayant d'exacts correspondants argiens ${ }^{34}$. Que tirer de cette identité de structure?

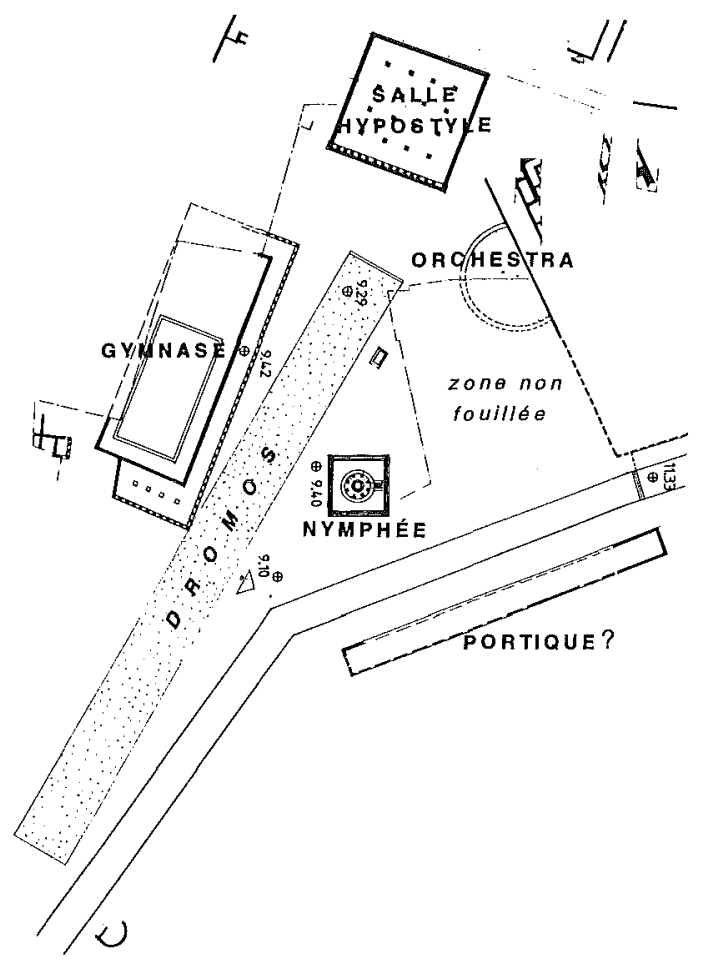

Fig. 1 : Principaux édifices du «Dromos» argien.

\section{Le Dromos dans la vie de la cité}

Il est patent que la Sparte impériale visitée par Pausanias était un musée, où l'on avait ressuscité d'anciennes coutumes censées se référer aux institutions de Lycurgue, comme le jeu des Sphaireis, les gymnopédies, les flagellations 
d'Artémis Orthia, les courses et les Dioskoureia, à côté d'autres rituels qui avaient survécu, au premier rang desquels les Hyacinthies ${ }^{35}$.

Comme le Dromos est le lieu de convergence absolu de la description de Pausanias, on pensera sans risque qu'il devait être le foyer ancestral, le cour de la cité primitive, un lieu éminent de la vie civique. Comment toutefois ranimer à partir de Pausanias la vie du Dromos aux époques hautes? Il faudrait être en mesure de faire la part des éléments neufs introduits à l'époque impériale, comme le sont manifestement les références aux éphèbes et à l'éphébie, l'organisation de celle-ci par ôbès ${ }^{36}$ et les bâtiments identifiés comme gymnases $^{37}$. L'éphébie d'époque impériale ne reproduit certainement plus les étapes de l'agôgé primitive ${ }^{38}$. Tout effort pour superposer les éléments livrés par Pausanias et ceux transmis par Xénophon dans sa République des Lacédémoniens paraît a priori voué à l'échec. Tenant compte toutefois, d'une part, de l'importance morale que conserve à l'époque impériale la référence à Lycurgue $^{39}$ et, d'autre part, de la structure particulière du Dromos autant que de la destination de l'Aphétaïde, il n'est pas désespéré de repérer dans les éléments fournis par Pausanias ce qui constitue une référence «active » au passé. À cet égard, deux particularités du Dromos ressortent singulièrement :

1. Il apparaît d'abord comme un ensemble religieux articulé à une piste de course, l'Aphétaïde, vouée à des concours matrimoniaux.

2. Le passage de l'éphébie à l'«âge adulte », ou si l'on veut à la catégorie des andres, est marqué par des compétitions spéciales, qui valent à ceux qui y participent «par ôbè » d'être appelés Sphaireis ${ }^{40}$. Il s'agit d'un agôn spécifique qui mettait aux prises les ôbès représentées en la circonstance par un certain nombre d'éphèbes. Jeu de balle ou combat de boxe ${ }^{41}$, peu importe ici. Il suffit de constater qu'il s'agit d'un rite de passage, important à en juger par la quinzaine d'inscriptions qui célébraient les vainqueurs du concours.

35 Sur les fêtes spartiates et leur horizon culturel, en plus des ouvrages et articles cités ci-dessus n. 30, voir H. JEANMAIRE, Courot et Courètes, 1939, qu'on peut actualiser par CARTLEDGE \& SPAWFORTH, op. cit. (n. 4), p. 190-211, et M. PETTERSSON, Cults of Apollo at Spaita, 1992.

36 Cf. CARTLEDGE \& SPAWFORTH, op. cit (n. 4), p. 206.

37 Le seul dont la consécration nous soit connue est attribué à Euryclès, sans aucun doute l'ultime représentant d'une richissime «dynastie » locale d'époque impériale, cf. ibid., p. 129-130.

38 Une comparaison de l'agôgè spartiate avec l'éphébie n'en avait pas moins été argumentée e.a. par R.F. WILLETTS, Aristocratic Society in Ancient Crete, 1955, p. 110-118.

39 Au point de le retrouver honoré, en compagnie d'Héraklès, comme « dieu fondateur » dans une inscription du IIe s. ap. J.-C., cf. A.J.S. SPAWFORTH, in $A B S A, 89$ (1994), p. 438. On ne peut que relier cette association des archégètes de Sparte aux statues d'Héraklès et de Lycurgue qui flanquaient les deux accès au Plataniste (PAUS., III, 14,8).

40 Sur la sphairomacbia, cf. CARTLEDGE \& SPAWFORTH, op. cit. (n. 4), p. 203-207; HATZOPOUlos, op. cit. (n. 30), p. 90, qui renvoie à un mémoire inédit sur Le Culte des Dioscures et la double royauté à Sparte (1970), et PETTERSSON, op. cit. (n. 35), p. 47.

41 Pour le sens du mot $\sigma \phi \alpha \uparrow \rho \alpha, c f$. notamment J. DELORME, BCH, 106 (1982), p. 67-68. 
Pour comprendre l'une et l'autre caractéristiques et saisir leur profondeur historique, on ne manquera pas de rapprocher le Dromos spartiate du terme dromeus connu par les inscriptions crétoises ${ }^{42}$. Et nous y sommes d'autant plus invités que l'épiclèse Dromaios d'Apollon est commune aux Crétois et aux Spartiates, que, d'autre part, l'intervention du sage crétois Épiménidès ${ }^{43}$ dans la constitution de Sparte est incontestable ${ }^{44}$. Si les éxégètes des institutions crêtoises ne s'accordent pas dans le détail sur l'interprétation du terme «dromeus », il ne fait toutefois aucun doute que l'octroi du titre impliquait un moment important dans la vie du citoyen crétois ${ }^{45}$. La différence entre apodromos et dromeus dans le code des Lois de Gortyne se situant au niveau du mariage - le code prévoit le cas d'exception où l'apodromos peut se marier $^{46}$-, on peut même conclure que le titre dromeus impliquait l'accès du jeune au mariage. Il implique aussi ${ }^{47}$ l'accès aux courses que l'on devait pratiquer dans le Dromos et à la vie collective de l'andreion au sortir de l'«éphébie ». Relevons enfin l'obligation qui était faite aux jeunes Crétois, membres d'une même agela, de se marier collectivement ${ }^{48}$. D'où l'on peut conclure que le dromeus est, à l'instar des Dioscures, un citoyen à part entière, sorti d'initiation, normalement marié et admis au Dromos.

Si de Crète on revient alors à Pausanias, on ne manquera pas de relever que l'Aphétaïde, encore à l'époque impériale, est le lieu symbolique d'une course nuptiale, celle des prétendants de Pénélope, dont l'institution est certainement ancienne. Pausanias l'attribue à Icarios, qui aurait en cela imité l'argien Danaos, référence qui nous renvoie, en cascade, à la description que donne Pindare ${ }^{49}$ d'un concours exactement similaire, exécuté dans une autre ville dorienne, particulièrement proche de Sparte mais aussi d'Argos : Cyrène.

42 Cf. WILlETTS, op. cit. (n. 38), p. 11-14, et Ancient Crete. A Social History, 1965, p. 113.

43 Cité deux fois par PAUSANIAS, III, 11, $11 \& 12,11$. On éclairera les commentaires de Pausanias par le long passage de DIOGËNE LAËRTE, I, 109-115, cf. G. COLLI, La Sagesse grecque, II, p. $54-59$ (p. 55 , en note, sont données toutes les références aux éditions de fragments où ce texte essentiel est reproduit).

44 Et l'on pourrait aisément élargir le parallèle, par ex. en cherchant aussi en Crète et autour d'Ariane les antécédents du Choros, comme le propose S.H. LONSDALE, Dance and Ritual Play in Greek Religion, 1993, p. 114-121. Dans le même ordre d'idées, A. LEMBESSI, in BCH 115 (1991), p. 99-123, tire parti de trouvailles crétoises pour expliquer le rite de flagellation pratiqué à Sparte.

45 Récemment, HATZOPOULOS, op. cit. (n. 30), p. 59 \& 88, a remis l'institution en perspective, en élargissant la comparaison à la Macédoine où les découvertes récentes ouvrent des champs nouveaux à la connaissance de l'hellénisme.

46 VII, 35-44 not., cf. R.F. WILLETTS, The Law Code of Gortyn, 1967, p. 23-25, et op. cit. (n. 38), p. 11-12. C'est pour nous, ici, une question secondaire que les classes d'âge de l'agôgè spartiate ne soient pas encore fixées avec précision, voir WILLETTS, op. cit. (n. 38), p. 7 sq., et dernièrement PETTERSSON, op. cit. (n. 35), p. 78-90.

47 Comme nous l'apprend un commentaire d'Aristophane de Byzance conservé par EUSTHATE, 1592, 58 (ad. o 172), $c f$. WILlETTS, op. cit. (n. 38), p. 11.

48 StRABON, X, 482, cf. ibid., p. 8.

49

Pythiques, IX, 105-125. 
Le concours nuptial institué par Antée - là aussi à l'imitation de Danaos -, décrit dans la IXe Pythique, peut être ainsi résumé : les jeunes filles à marier étaient placées dans le chœur et attendaient qui le premier toucherait leurs vêtements au terme de la course. On y retrouve l'organisation caractéristique d'un mariage collectif comme celui des agelai crétoises. Et l'on sait qu'à Sparte, comme en Crète, il était mal venu de refuser le mariage, le passage d'un état à l'autre étant « impératif $»^{50}$.

L'intérêt du parallèle tient à ce que les institutions de Cyrène, colonie de Théra et l'un des grands centres du culte d'Apollon Carneios, ne peuvent que dériver de la mère patrie, Sparte. Qu'un concours nuptial à la course y soit attesté par Pindare suffit à montrer que le concours matrimonial spartiate est ancien. Or, à Sparte comme à Cyrène, son invention est attribuée à l'argien Danaos, référence qui prend tout son sens lorsque l'on considère l'« agora » argienne fouillée par l'École française : le rituel même de la «course des prétendants » $y$ trouve une illustration directe dans la disposition des lieux ${ }^{51}$.

À Sparte, la proximité du départ de l'Aphétaïde et du local des Bidiéens chargés de la surveillance des concours d'éphèbes invite à faire de la « course » de l'«Aphétaïde » à l'époque impériale, celle que l'on disait « la course des prétendants », une course d'éphèbes. Il n'est guère hasardeux de la relier à l'autre épreuve imposée aux éphèbes, l'agôn de la Sphaira à la suite duquel - précise Pausanias - les éphèbes devenaient andres. Il est vraisemblable que le terme «sphaireus» employé à l'époque impériale soit, d'une manière ou d'une autre, l'équivalent de l'ancien titre «dromeus». Interpréter le terme en référence au mariage impliqué dans l'accession à la classe des andres paraît, en effet, inévitable.

\section{Les dieux du Dromos}

Que dans les cités doriennes le «Dromos» ait été un lieu d'intégration des nouveaux citoyens implique que les dieux qui y étaient présents ou qui en étaient voisins y fussent vénérés en liaison avec la formation de ces mêmes citoyens, que chaque divinité devait y incarner un aspect particulier des valeurs que l'on entendait inculquer à la jeunesse, que chacune participait d'un univers mental, caractéristique de l'éducation dorienne, du moins pour ce qui concerne les agoras vouées à Apollon Carneios. Il n'est donc pas étonnant de retrouver sur les agoras doriennes les mêmes dieux et héros, tels les Dioscures, Héraklès, Poseidon, Dionysos, Aphrodite, Héra, Artémis, dans des attributions largement identiques - d'un site à l'autre on ne perçoit guère plus que des « nuances », dues davantage à l'action du temps et à des circonstances particulières qu'à une différence de nature. Chacun tenait un rôle très précis, dont le décryptage est

PLUTARQUE, Lycurgue, 15, 1-3.

51 Voir l'étude de $B C H, 118$ (1994), p. 152-153. 
moins difficile qu'il pourrait paraître. L'intérêt de les saisir dans le complexe du Dromos vient de ce que cela nous oblige à les étudier dans leurs interactions et leur complémentarité.

Nous ne pouvons ici développer ce point de vue. Nous nous contenterons d'illustrer le propos en attirant l'attention sur les divinités les plus caractéristiques, à savoir les Dioscures, Déméter et Corè, Dionysos et Poseidon. Que les Dioscures soient les patrons de l'Aphétaïde n'a pas besoin de démonstration : Pausanias nous l'apprend sans détour. Leur position en fait donc les patrons des courses nuptiales, les prototypes du mariage. On ne manquera pas de relever qu'à Argos, les Dioscures patronnaient des courses au Ve siècle et qu'ils étaient représentés dans leur temple en compagnie de leurs épouses et de leurs fils ${ }^{52}$.

Autour de Déméter et Corè s'organisaient d'autre part les « initiations ». Ici encore si l'on compare Sparte et Argos, la description de Pausanias est suffisamment claire pour qu'on retrouve les divinités dans leur aspect familier. Les sanctuaires contigus de Déméter Chthonia et Corè Sôteira, tous deux fondés par Orphée et proches d'Aphrodite Olympienne, situent les deux déesses vénérées à Sparte dans une ambiance «éleusinienne ». Le téménos argien de Déméter et Corè est, lui, en étroite consonance avec le monde infernal ${ }^{53}$. Comme celle de Sparte, la Déméter de l'agora argienne est avant tout une déesse «chthonienne », qu'on ne manquera pas de mettre en relation avec l'institution des Thesmophories par les Danaïdes ${ }^{54}$. Que de part et d'autre, Déméter ait été l'occasion de célébrer des "mystères », nous n'en douterons pas. Le Dromos argien reproduit en effet la même théorie de divinités que celles vénérées à Lerne ${ }^{55}$ - Déméter-Corè, Dionysos et Poseidon - et nous savons que Lerne était un lieu où l'on accomplissait des mystères. Pour Sparte, les données sont tout aussi nettes, bien que spécifiques : si le Dromos est dominé par les Dioscures, il est aussi sacralisé par Apollon Carneios-Oikétas, dont le culte est conjoint, dans les prêtrises communes d'époque impériale, à celui d'Héraklès, de Poseidon, mais surtout de Corè. On ne peut manquer de souligner cette fois le parallélisme qui s'instaure avec les divinités du bois sacré d'Apollon Carneios à Andania ${ }^{56}$ : on y retrouve, en effet, les Dioscures en Mégaloi Théoi, Déméter et Corè/Hagnè, Hermès psychopompe - et Apollon Carneios. La réalité des mystères d'Andania rend compte de la présence de Déméter et Corè au sein du Dromos spartiate et l'on

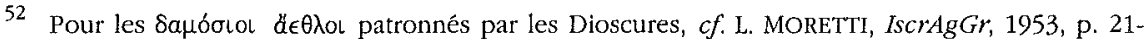
22, n 10, et P. AMANDRY, Éfudes argiemnes, 1980 (BCH Suppl. VI), p. 211 n. 3. Pour leur temple et le groupe statuaire, $c f$. A. HERMARY, in LIMC, III 1 (1986), p. 589-590.

53 PAUS., II, 22, 1-4. Sur cela, cf. Le Nymphée de l'agora d'Argos, p. 217-220.

54 HÉRODOTE, II, 171

55 Pour les sanctuaires et dieux de Leme, $c f$. dernièrement G. CASADIO, Storia del culto di Dioniso in Argolide, 1994, p. 223-325. La présence de Dionysos y est essentielle.

56 PAUS., IV, 33, 4-6. 
ne manque pas de relever de part et d'autre leur identique association à Apollon Carneios.

Nous en resterons là, non sans souligner, pour conclure, tout l'intérêt qu'il peut y avoir à aborder l'étude des dieux grecs dans le contexte très concret du Dromos et de l'agora doriens, en tout état de cause dans leurs relations réciproques au sein d'un univers mental global.

Facultés Universitaires N.D. de la Paix

Patrick MARCHETTI

61 , rue de Bruxelles

B - 5000 NAMUR 